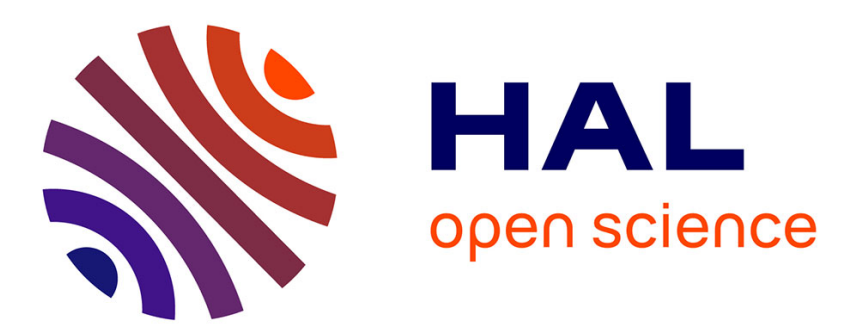

\title{
Compact Cold-Atom Clock for Onboard Timebase: Tests in Reduced Gravity
}

Mehdi Langlois, Luigi de Sarlo, David Holleville, Noel Dimarcq, Jean-François Schaff, Simon Bernon

\section{- To cite this version:}

Mehdi Langlois, Luigi de Sarlo, David Holleville, Noel Dimarcq, Jean-François Schaff, et al.. Compact Cold-Atom Clock for Onboard Timebase: Tests in Reduced Gravity. Physical Review Applied, 2018, 10 (6), pp.064007. 10.1103/PhysRevApplied.10.064007 . hal-02050966

\section{HAL Id: hal-02050966 https://hal.sorbonne-universite.fr/hal-02050966}

Submitted on 27 Feb 2019

HAL is a multi-disciplinary open access archive for the deposit and dissemination of scientific research documents, whether they are published or not. The documents may come from teaching and research institutions in France or abroad, or from public or private research centers.
L'archive ouverte pluridisciplinaire HAL, est destinée au dépôt et à la diffusion de documents scientifiques de niveau recherche, publiés ou non, émanant des établissements d'enseignement et de recherche français ou étrangers, des laboratoires publics ou privés. 


\title{
Compact Cold-Atom Clock for Onboard Timebase: Tests in Reduced Gravity
}

\author{
Mehdi Langlois, ${ }^{1,{ }^{*}}$ Luigi De Sarlo, ${ }^{1}$ David Holleville, ${ }^{1}$ Noël Dimarcq, ${ }^{1}$ Jean-François Schaff, ${ }^{2}$ and \\ Simon Bernon ${ }^{3}$ \\ ${ }^{1}$ SYRTE, Observatoire de Paris, PSL Research University, CNRS, Sorbonne Universités, UPMC Univ. Paris 06, \\ LNE, 61 avenue de l'Observatoire, 75014 Paris, France \\ ${ }^{2}$ Muquans, Institut d'Optique d'Aquitaine, rue François Mitterand, 33400 Talence, France \\ ${ }^{3} L P 2 N$, Institut d'Optique d'Aquitaine, Université Bordeaux, CNRS, 33400 Talence, France
}

(Received 15 June 2018; revised manuscript received 16 August 2018; published 4 December 2018)

\begin{abstract}
We present a compact atomic clock using cold rubidium atoms based on an isotropic light cooling, a Ramsey microwave interrogation, and an absorption detection. Its technology readiness level is suitable for industrial transfer. We use a fiber optical bench, based on a frequency-doubled telecom laser. The isotropic light cooling technique allows us to cool down the atoms in $100 \mathrm{~ms}$ and works with a cycle time of around $200 \mathrm{~ms}$. We carry out measurements in simulated microgravity and obtain narrow fringes, up to $1.25 \mathrm{~Hz}$.
\end{abstract}

DOI: 10.1103/PhysRevApplied.10.064007

\section{INTRODUCTION}

It is well known that global navigation satellite systems (GNSSs) such as a global positioning system (GPS) can provide synchronization to UTC better than $40 \mathrm{~ns}$. This limit, however, is typically reached only for a stationary platform with a calibrated receiver. For a moving platform, the timebase provided by the GNSS is subject to more systematics, including service availability and reliability. Furthermore, there is an increasing number of platforms for which high-accuracy inertial navigation is required and a GNSS is not an option. Examples of these platforms are submarines and deep-space missions. Last but not least, a highly reliable and accurate timebase can be used to upgrade the existing facilities on board the satellites of GNSS constellations.

The key ingredient of autonomous timebase generation is an oscillator, which can provide an intrinsically high stability $\left(1 \mu\right.$ s over one year or $3 \times 10^{-14}$ of relative instability [1]). This kind of performance is currently available only using hydrogen masers, which have indeed been miniaturized and constitute the main timebase generation on board the satellites of the GALILEO European GNSS.

Cold-atom-based atomic clocks currently realize the most accurate primary frequency standard in several metrology institutes worldwide [2] and will also be on board the International Space Station thanks to the PHARAO clock [3].

Despite these great achievements, no on-board cold-atom-based clock capable of achieving similar

\footnotetext{
*m.langlois@bham.ac.uk
}

performances to hydrogen masers in an easier configuration than the PHARAO clock has been demonstrated.

In this paper, we describe a compact atomic clock based on cold ${ }^{87} \mathrm{Rb}$ atoms, which will fill this gap. To this end, we report on the clock operation on board an airbus A300 performing parabolic flights and we analyze the limits of the clock in the present and ideal configurations for operation on board in both standard and reduced gravity. We compare the results obtained to those on the ground.

\section{SETUP}

\section{A. Approach}

Our setup is a full redesign of our previous experiment [4], with the following major differences: we now use ${ }^{87} \mathrm{Rb}$ atoms instead of ${ }^{133} \mathrm{Cs}$ and the laser system is based on frequency-doubled telecom technology [5], allowing very compact and robust systems. The electronics, vacuum chamber, and magnetic shielding are also renewed and adapted to operation on board.

Using ${ }^{87} \mathrm{Rb}$ is favorable for two reasons: the coldcollision shift is reduced compared to ${ }^{133} \mathrm{Cs}$ [6], allowing improved long-term stability, and it allows the use of compact fiber lasers.

\section{B. Laser system}

Our new laser system, produced by the company Muquans, is based on two fiber telecom external-cavity diode lasers operating at $1560 \mathrm{~nm}$, which are then frequency-doubled to reach the rubidium lines around 780 $\mathrm{nm}$. The first (master) is frequency-doubled and locked by absorption spectroscopy to the ${ }^{85} \mathrm{Rb}\left|5 S_{1 / 2}, F=3\right\rangle \rightarrow$ 
$\left|5 P_{3 / 2}, F^{\prime}=3,4\right\rangle$ crossover. A slave laser is phase locked to the master with an offset frequency of approximately $620 \mathrm{MHz}$ provided by a direct digital synthesizer (DDS), allowing the dynamical change of the frequency during the different stages. The repumper frequency is generated by a phase modulator operating at approximately $6.6 \mathrm{GHz}$. Power is provided by an erbium-doped fiber amplifier. An acousto-optic modulator is used to reduce or turn off the power. A second frequency-doubling crystal is used after amplification, and a free-space splitter divides the power into two fibers (one for the cavity beams, and one for cooling and detection along the vertical direction). The power ratio is optimized to approximately $30 \%$ in the vertical beam with a waveplate to maximize the atom number. Inside the splitter, mechanical shutters are placed on the two paths to provide attenuation higher than $120 \mathrm{~dB}$ during Ramsey spectroscopy. The total output power is always $>120 \mathrm{~mW}$ at $780 \mathrm{~nm}$. This full laser system (optics and electronics) is fully integrated into a 19-in. wide, 4 U-high rack.

\section{EXPERIMENTAL CYCLE}

As in our previous work, cooling, state preparation, interrogation, and detection are carried out in a glass cell positioned at the center of a spherical copper cavity. This is illustrated in Fig. 1.

\section{A. Cooling}

First, atoms are cooled by isotropic light cooling [7-9], the light being injected in the cavity by six single-mode fibers (not in Fig. 1). A 100-ms-long Doppler cooling $[10,11]$ is performed with about $120 \mathrm{~mW}$ of total power, detuned by $13.6 \mathrm{MHz}(2.24 \Gamma)$ from the ${ }^{87} \mathrm{Rb}$ cycling transition. Repumping is obtained by phase modulation. This phase is followed by a 2-ms-long sub-Doppler cooling stage [12]. In this process, the laser beam is detuned by $58.4 \mathrm{MHz}(9.6 \Gamma)$ and the overall power is reduced by $40 \%$. We also reduce the repumper intensity. At the end of the cooling stage, we have approximately $2 \times 10^{7}$ atoms in all magnetic sublevels at a temperature around $10 \mu \mathrm{K}$.

\section{B. Depumping}

State preparation is achieved by tuning the laser to the $F=2 \rightarrow F^{\prime}=2$ transition without repumper for $2 \mathrm{~ms}$. Only the atoms in the $F=1, m_{F}=0$ magnetic sublevel will interact with the microwave, the other transitions being Zeeman-shifted by the constant magnetic field of $10 \mathrm{mG}$.

\section{Interrogation}

We work with a Ramsey interferometer scheme [13] in a $\pi / 2-\pi / 2$ configuration. The microwave, which is resonant with the $F=1, m_{F}=0 \rightarrow F=2, m_{F}=0$ clock
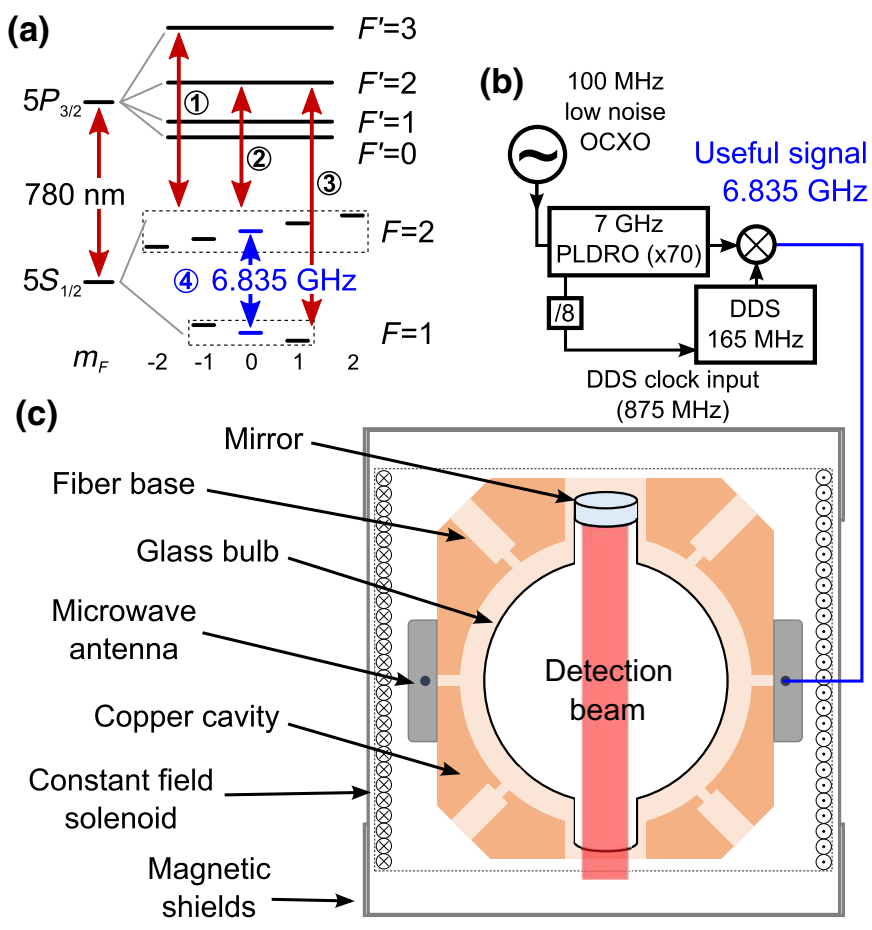

FIG. 1. Experimental setup. (a) Optical transitions involved in the detection and preparation of the cold sample with isotropic cooling (red): (1) cooling is performed red-detuned from the cycling transition, detection is done on resonance, (2) depumping to $F=1$ is achieved on the $2 \rightarrow 2$ transition, (3) repumping light is obtained by phase modulation, (4, blue) microwave clock transition. (b) The clock frequency $v_{c} \simeq 6.835 \mathrm{GHz}$ is generated from a $100 \mathrm{MHz}$ oven-controlled crystal oscillator (OCXO), which is multiplied by 70 and then mixed to a signal generated by a DDS. This signal is injected through one of the two microwave antennas. (c) The glass bulb is within a copper cavity, which is enclosed in a solenoid generating a constant magnetic field. All these components are isolated from external magnetic fields by magnetic shields.

transition of ${ }^{87} \mathrm{Rb}$, is injected into the cavity through one antenna. At this frequency, the cavity sustains a cylindrical $\mathrm{TEM}_{011}$ mode with a quality factor $Q=6636$. To get the maximal stability of its resonance frequency, we control its temperature with a coaxial heating cable wound around it. The microwave pulses are achieved by switching the microwave signal. This gives an atomic phase shift according to the frequency difference between the atoms' clock transition and the local oscillator (LO) [see Eq. (1)].

\section{Detection}

Finally, absorptive detection is performed on resonance with a photodiode and without the repumper, with the vertical beam only. The intensity is close to $I_{\text {sat }} / 100$ to prevent any saturation effect (for the ${ }^{87} \mathrm{Rb} D_{2}$ transition, $I_{\mathrm{sat}} \simeq 1.67 \mathrm{~mW} / \mathrm{cm}^{2}$ ). A second photodiode measuring the laser's incident intensity is used to normalize the 
(a)

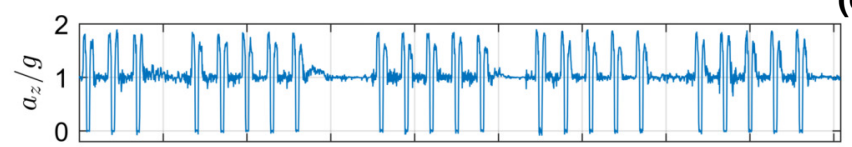

(b)

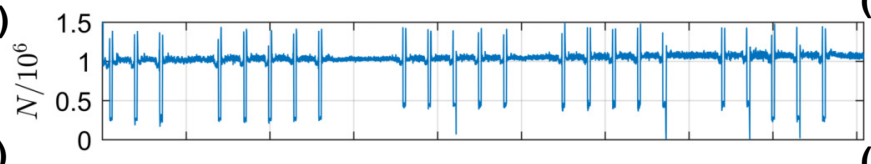

(c)

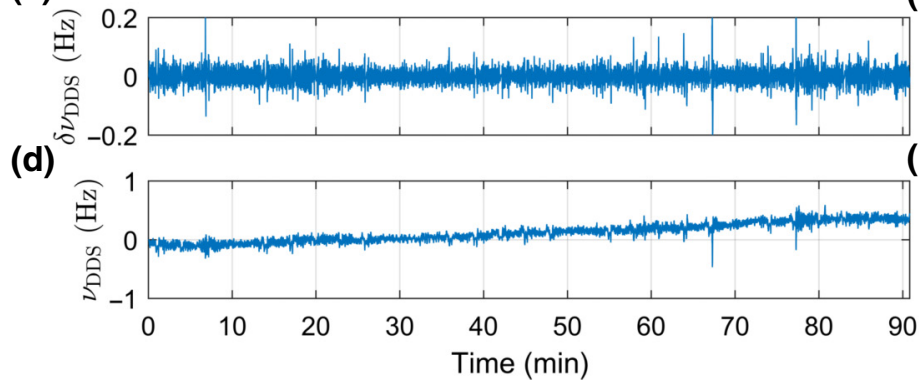

(e)

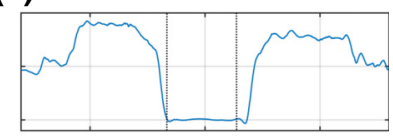

(f)

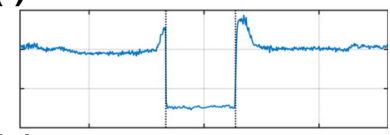

(g)

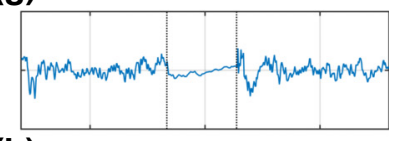

(h)

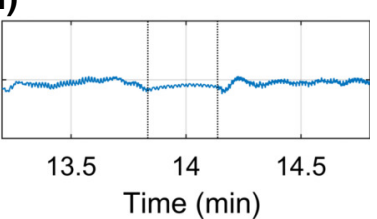

FIG. 2. LO locked to the atomic signal during the entire flight. (a) Vertical acceleration in units of $g=9.81 \mathrm{~m} / \mathrm{s}^{2}$. (b) Atom number $N$. (c) Frequency correction applied to the $\mathrm{LO}$ at $6.835 \mathrm{GHz}$ (through the DDS, see Fig. 1 and text). (d) DDS frequency (offset of approximately $165 \mathrm{MHz}$ removed). (e)-(h) Enlargement of the fourth parabola. absorption signal and improve the signal-to-noise ratio [14]. This allows the measurement of the number of atoms remaining in $F=2$ after the microwave interaction.

\section{E. Clock operation}

In this article, we present results obtained either by scanning experimental parameters (LO frequency, Ramsey time, and so on) or in closed-loop operation. In this latter case, the LO is stabilized to the Rb clock frequency by alternately measuring the atom number at "half-fringe" on either side of the central fringe (frequency $v \simeq v_{c} \pm$ $\Delta v / 2)$. The difference between two consecutive measurements gives an error signal used to correct the LO frequency. Feedback is applied to the DDS frequency, not directly to the OCXO, which remains "free" (its frequency may drift). The correction signal and the atom number are shown in Fig. 2, where the LO is locked to the atomic resonator during an entire flight.

\section{ISSUES}

Close to resonance, the interference signal is well approximated by

$$
N(v)=N_{1 / 2}\left[1+C \cos \left(\pi \frac{v-v_{c}}{\Delta v}\right)\right],
$$

where $N_{1 / 2}$ is the atom number at half-fringe, $C$ is the contrast of the interference pattern, $v$ is the LO frequency, $v_{c}$ is the ${ }^{87} \mathrm{Rb}$ clock frequency, and $\Delta v \simeq 1 /\left(2 T_{R}\right)$ is the FWHM of the central Ramsey fringe. The Ramsey interrogation time is $T_{R}$. In closed-loop operation, the clock's fractional frequency stability [15] is related to the signal, noise, contrast, Ramsey time, and cycle duration $T_{C}$ by

$$
\sigma_{y}(\tau)=\frac{1}{\pi} \frac{\Delta v}{v_{c}} \frac{1}{\operatorname{SNR}} \sqrt{\frac{T_{C}}{\tau}},
$$

$\tau$ being the integration time. Here, the relevant signalto-noise ratio is $\mathrm{SNR}=C N_{1 / 2} / \delta N_{1 / 2}$, where $\delta N_{1 / 2}$ is the noise at half-fringe, which is well estimated by the first point of the Allan deviation of the atom number at half-fringe. For all the data presented, the cycle time is $T_{C}=T_{R}+130 \mathrm{~ms}$ (sum of the cooling, preparation, and detection times). Therefore, for a given species and transition, the clock is optimized by maximizing the ratio $\mathrm{SNR} \times T_{R} / \sqrt{T_{C}}$.

On the ground, we found this optimum for cooling and Ramsey times close to $100 \mathrm{~ms}$ and $T_{R}=40 \mathrm{~ms}$, respectively (see Fig. 4, red stars). This duration of the cooling stage is a compromise between gathering enough atoms to be shot-noise limited [4], i.e., having a good SNR, and not making the cycle time too long. The optimum of $40 \mathrm{~ms}$ is related to the free fall of the atoms in the cavity (corresponding to approximately $8 \mathrm{~mm}$ of displacement). When this time becomes longer, the contrast drops dramatically. The temperature is on the order of $10 \mu \mathrm{K}$ and does not limit significantly on the ground.

In weightlessness, one can expect to increase $T_{R}$ significantly, therefore improving the stability [see Eq. (2)].

If we increase the interrogation time $T_{R}$ we reduce the FWHM of the fringes $\Delta v$ and obtain a better frequency discrimination.

For instance, longer Ramsey times tend to improve the stability because of the narrower fringe, but to worsen it because of the reduction of the atom number and contrast.

On Earth we are limited by the falling time of the atoms, which leads to dramatic loss of atoms and contrast for an interrogation time longer than $60 \mathrm{~ms}$. So we have made measurements under microgravity.

\section{A. Parabolic flights: the environment}

The Rubiclock experiment participated in two parabolic flight campaigns on board the Airbus A300 ZERO-G. 
These campaigns were funded by CNES and organized by Novespace. Each campaign consisted of three flights and each flight included 31 parabolas. Thus a whole campaign provides $31 \mathrm{~min}$ of microgravity, but is sliced into 93 slices of $20 \mathrm{~s}$. Each microgravity slice is framed by two $20 \mathrm{~s}$ slices of $2 g$ hypergravity, all separated by a $1 g$ phase lasting at least $1 \mathrm{~min}$. Under these conditions, it is not possible to integrate the atomic signal more than $20 \mathrm{~s}$ at a time during the $0 g$ or $2 g$ phases.

During the microgravity phases, the residual acceleration felt by the setup depends on its location in the plane, and of course on the parabola quality. The highest fluctuations are in the direction of the local vertical of the aircraft, and are typically about $0.01 \mathrm{~g}$ RMS with oscillations of $\pm 0.05 g$ peak values increasing up to $+0.1 \mathrm{~g}$ at the beginning and at the end of the parabola. As the detection beam of the setup is only $7 \mathrm{~mm}$ diameter, horizontal acceleration fluctuations have a significant effect on the detected atom number. Therefore, the SNR of the atomic signal is all the more limited by these fluctuations as the Ramsey interrogation time is long.

We manage to operate the clock continuously during the parabolic flight that included the parabolas, wild movements into the Earth's magnetic field, and a lot of jolts.

\section{B. Measurements in microgravity}

A trade-off has to be found on the Ramsey time, to optimize the clock frequency stability, which is proportional to $1 /\left(T_{R} \times \mathrm{SNR}\right)$. Measurements performed during the first campaign show that the optimized Ramsey time for $0 \mathrm{~g}$ phases is between 100 and $200 \mathrm{~ms}$. Above $200 \mathrm{~ms}$, the SNR is quickly degraded by the fluctuations of the acceleration of the plane. If installed on board less noisy carriers, such as uninhabited satellites, this clock could greatly improve its metrological performance with Ramsey times of up to $500 \mathrm{~ms}$.

During a first campaign we carried out measurements of atom recaptures and measurements of Ramsey fringes with long interrogation time. The phenomenon of recapture is increased in microgravity because the atomic cloud does not fall but thermally expands. We can recapture atoms that remained in the cavity, which allows us, for the same cooling time, to get more atoms.

Regarding the interrogation time we reached $400 \mathrm{~ms}$ with a contrast of $56 \%$, as we can see on Fig. 3a. This equates to a $1.25 \mathrm{~Hz}$ fringe FWHM. While on Earth we cannot go further than $70 \mathrm{~ms}$ with a contrast of $29 \%$. This represents a good result for an on-board clock, considering the vibration noise aboard the aircraft.

We participated in a second flight campaign, during which we activated the frequency feedback loop to keep the LO frequency on the rubidium clock frequency. This feedback loop performs an integration over five points.

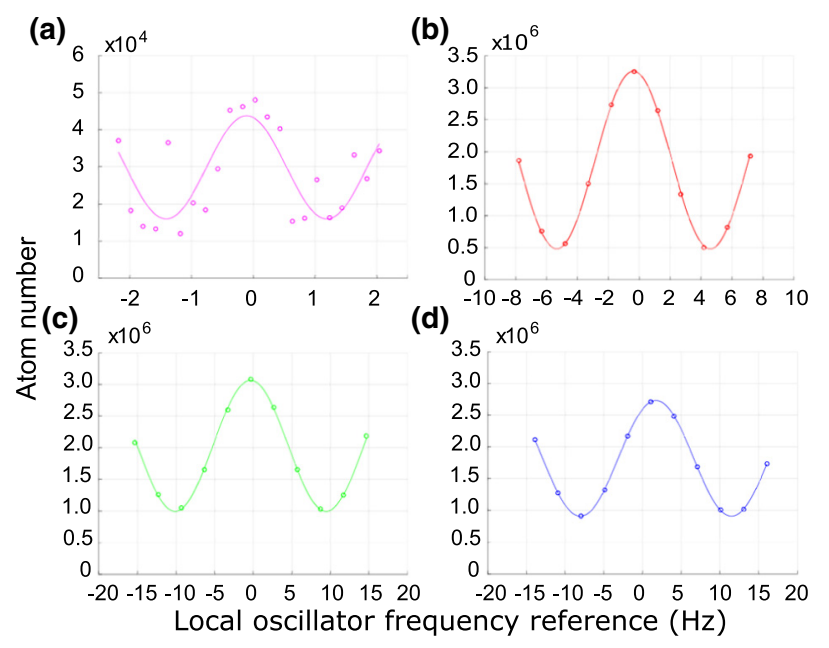

FIG. 3. Fringes for different interferometry times and different environments. (a) The fringe at $400 \mathrm{~ms}$ in $0 \mathrm{~g}$. (b) The fringe at $100 \mathrm{~ms}$ in $0 g$. (c) The fringe at $50 \mathrm{~ms}$ in $1 g$ on board. (d) The fringe at $50 \mathrm{~ms}$ in $1 \mathrm{~g}$ on the ground.

First we tested the clock capacity to stay locked during a flight. During a flight the aircraft performs thirty-one $0 g$ phases, each framed by $2 g$ phases, all separated by $1 g$ phases. We modified the interrogation time between the $0 g$ phase and the other phases. The clock stayed locked on the rubidium frequency during all the phases $(310 \mathrm{~g}$ phases and $622 g$ phases, for more than $90 \mathrm{~min}$ ).

\section{Discussion of the results}

We now try to estimate the ultimate reachable short-term stability of the Rubiclock in weightlessness conditions. A direct measurement of the stability shows several issues:

(1) Integration time limited to $20 \mathrm{~s}$

(2) Absence of a reference metrological signal for direct comparison

(3) High sensitivity of the LO to vibration leading to a large increase of the Dick effect and degradation of the measured short-term stability

(4) Dependence of the atom number on the acceleration fluctuations of the carrier, leading to short-term stability degradation in a noisy environment such as on the aircraft, compared to those that can be reached in quiet conditions such as in a satellite.

Moreover, to keep the clock frequency locked on the $\mathrm{Rb}$ transition all through the $1 g, 2 g$, and $0 g$ phase successions, the feedback loop had to have a long time constant (approximately equal to $1 \mathrm{~s}$ ), which resulted in a slow frequency drift after each transition from $2 g$ to $0 g$ corresponding to a relaxation towards a new stationary state.

In this experiment, our LO is not optimized for the vibration; in an aircraft the clock stability is therefore limited by the LO stability because of the vibrations. However, some 
OCXO have been developed to be less sensitive to accelerations, such as the one for the Pharao clock [3]. We get rid of the noise due to the Dick effect [16] by performing our noise measurements at the top of the fringe. In this way we are only sensitive to the instrumental and quantum noises of the resonator. This gives us the ultimate stability that we can achieve with our clock or within a less noisy carrier, such as a satellite.

In order to compute the resonator short-term stability $\sigma_{y}(1 s)$, we measure the atom number vs the interrogation frequency, which we scan with the DDS, as shown in Fig. 5. Since we are limited by the parabola time (approximately $20 \mathrm{~s}$ ), we only take a few points (approximately 10) and perform a sine fit [see Eq. (1)]. For the noise, we make several measurements of the atom number at the top of the fringe (approximately 50). All this is done during the same parabola. This allows us to determine all the input parameters [see Eq. (5)]. The relevant SNR is the inverse of the atom-number-relative Allan deviation ( $a d e v$ ) for a delay of one experimental shot [see Eq. (3)]. This is because the error signal of the clock feedback loop is computed from two consecutive experimental shots, such that noise occurring at a higher time delay is rejected:

$$
\mathrm{SNR}_{1}=\frac{1}{\text { adev }}
$$

(a)

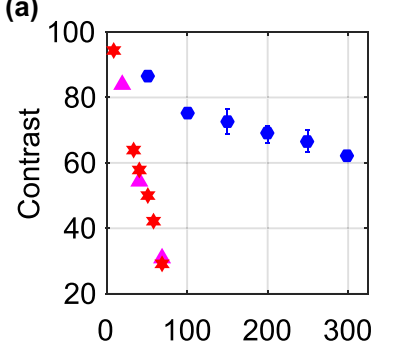

(c)

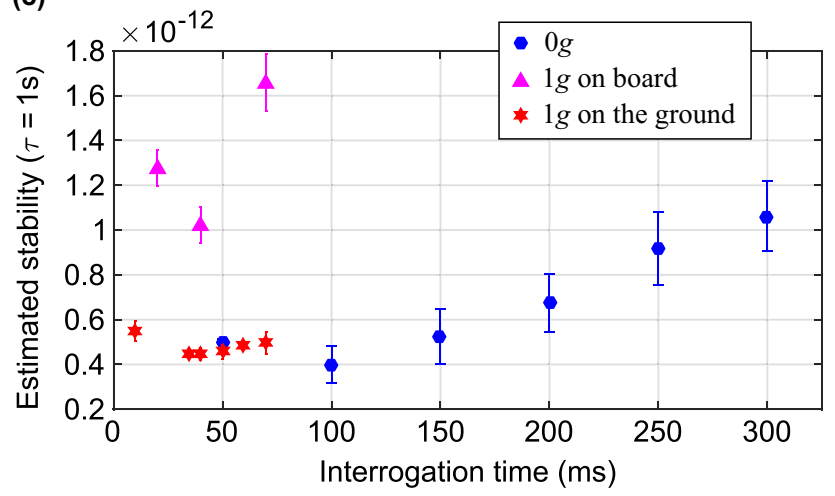

FIG. 4. At the top left the contrast of the interferometer. At the top right the SNR. At the bottom is the estimated stability at $1 \mathrm{~s}$. We perform several measurements for each time, and we plot the mean and the standard deviation of these values. The blue circles are the values in $0 g$. The purple triangles are the values in $1 g$ on board. The red stars are the values in $1 g$ on the ground.

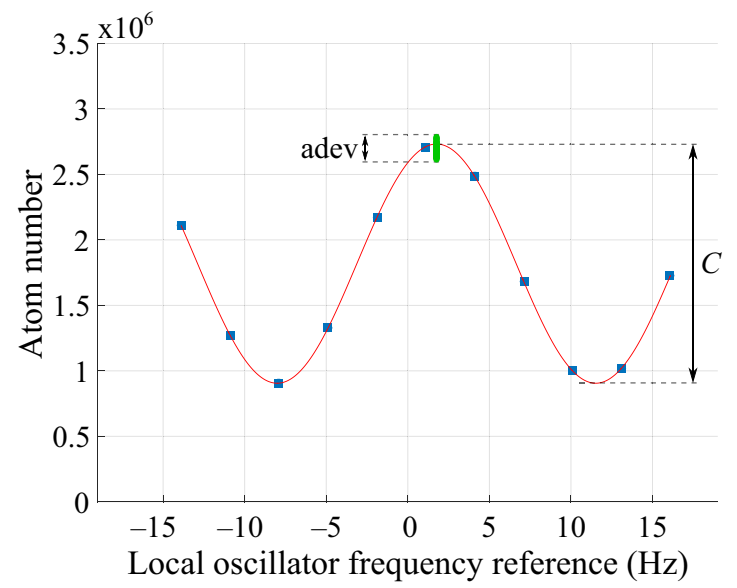

FIG. 5. Calculation of the resonator short-term stability. The blue squares are the atom number as a function of the interrogation frequency reference. The red line is the sine fit. The green circles are the atom number at the top of the fringe. With the sine fit, we compute the fringe contrast and with the atom number at the top of the fringe, we compute the Allan deviation.

As we said before,

$$
\mathrm{SNR}=C \mathrm{SNR}_{1}
$$

Considering Eq. (2), we can estimate the stability at $1 \mathrm{~s}$ with

$$
\sigma_{y}(1 s)=\frac{1}{\pi} \frac{1}{2 T_{R}} \frac{1}{v_{0}} \frac{\text { adev }}{C} \sqrt{T_{C}} .
$$

This expression neglects the shot noise and quantum projection noise, which are negligible compared to the noise we measure given our atom number. It also neglects the frequency noise of the LO and the Dick effect, which are both expected to be well below the noise we measure by performing the measurement at the top of the fringe.

The estimated stability is plotted in Fig. 4 for various experimental conditions $(0 g, 1 g$ on board and $1 g$ on the ground) and for $\tau=1 \mathrm{~s}$.

We carry out measures in $0 g$, and also during the $1 g$ phases on the aircraft to see the microgravity improvement. We perform measurements on the aircraft parked on the ground to observe the signal degradation brought about by vibration during the flight. We show on Fig. 4 the stability measurements at $1 \mathrm{~s}$, according to the atom interrogation time, as well as the contrast and the SNR.

In $0 g$, the blue circles have a maximum estimated stability around $100 \mathrm{~ms}$, with $3.8 \times 10^{-13}$ for the best measurement, but the points are quite dispersed. This is explained by the aircraft vibration, which induces displacement of the atoms during the interrogation time. The standard deviation of the position of the atoms in the quartz bulb is around $0.4 \mathrm{~mm}$ in the vertical axis and $1.6 \mathrm{~mm}$ in the radial axis. This is enough to bring out a few atoms of the 7 $\mathrm{mm}$ detection beam. The atom number depends strongly 


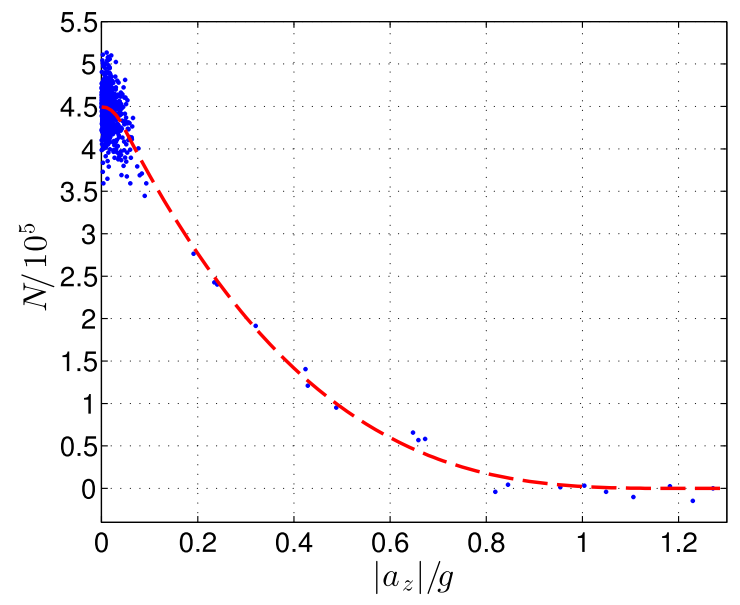

FIG. 6. Correlation between vertical acceleration and atom number in closed-loop operation.

on the vertical acceleration as we can see on Fig. 6, with variations due to aircraft vibrations. In $1 g$ on the aircraft, the purple triangles have a maximum stability around 40 $\mathrm{ms}$, with the best measurement at $9.1 \times 10^{-13}$, but on the ground with the same interrogation time the red stars have a stability at $1 \mathrm{~s}$ of $4.2 \times 10^{-13}$.

\section{Projection and comparisons}

In this experimental geometry, with a vertical detection beam, stability strongly depends on the horizontal vibration noise, even if the stability is still sufficient to achieve good performance. As we can see on Fig. 4, the $1 g$ on-theground measurements are more than twice as good as the $1 g$ on-board measurements, due to the aircraft vibrations, but the $0 g$ on-board measurements are still better than the $1 g$ on-the-ground measurements. Considering this, in a low-noise $0 g$ environment, such as a satellite, we can estimate that this clock will have a short-term stability at least 2 times better than on the aircraft. HORACE, the previous experiment based on the same architecture but with ${ }^{133} \mathrm{Cs}$ atoms, has a short-term stability of $2.2 \times 10^{-13}$, which is almost 2 times better than our clock on the ground, and a long-term stability of $4 \times 10^{-15}$ after $5 \times 10^{3} \mathrm{~s}$ of integration [4]. In the future, with more work to reduce the detection noise and identify technical noises during the interrogation (which is currently being undertaken), we can expect to reach better results due to the lower cold-collision shift of the ${ }^{87} \mathrm{Rb}[6]$ and further improve of the short-term stability. After consideration of these arguments, in a low-noise $0 g$ environment and reducing the noise budget as on HORACE, we can hope to reach in a satellite a short-term stability of around $10^{-13}$.

Other compact atomic clocks using different approaches have been developed. The miniature atomic clock [17], based on MEMS and optoelectronic devices, is more compact, but does not reach the same short-term stability,
$4 \times 10^{-10}$. The 5071A, a commercial clock based on a cesium-beam tube assembly [18], can reach a stability of $5 \times 10^{-12}$ in $1 \mathrm{~s}$ in a small volume. Another approach is to use a trapped-atom clock on a chip [19], which can reach a stability of $5.8 \times 10^{-13}$ in $1 \mathrm{~s}$. Clocks based on coherent population trapping [20] have a short-term stability of $3.2 \times 10^{-13}$ with a small head sensor $(<1 \mathrm{~L})$.

All these clocks are good instruments for a compact and transportable timebase. Due to its configuration, by isotropic light cooling [7-9] and cold atoms in free fall in a microwave cavity, the Rubiclock experiment is the clock that benefits most from the $0 g$ environment, as it allows increase of its interrogation time by keeping the same contrast [as shown in Fig. 4(a)] and increasing the recapture effect to reduce the cycle time.

\section{CONCLUSION}

In conclusion, we demonstrate the interesting shortterm stability of a compact and transportable atomic clock $(<100 l)$, its better performance under microgravity, and its ability to operate in a noisy environment. The clock is already in industrial development with the company Muquans. Its characteristics make it an interesting candidate for space clocks. Long-term stability measurements have begun to be carried out, to be compared with the frequency standard located in SYRTE.

\section{ACKNOWLEDGMENTS}

We are grateful to Bruno Desruelle and the Muquans team, Fabrice Tardif, Mathieu Guéridon, and Raphäl Bouganne, for their contribution to the laser system, control unit, and software, and assistance in designing the vacuum chamber. We would like to thank the electronic service of the SYRTE, Michel Lours, Laurent Volodimer, and José Pinto, the mechanical service of the SYRTE, Bertrand Venon, Florence Cornu, Stevens Ravily, and Louis Amand, the mechanical service of the GEPI, JeanPierre Aoustin, and the mechanical service of the LERMA, Laurent Pelay, for the great support they provided us during the setting up of the clock and the parabolic flight campaign. We want to thank the CNES, Jérôme Delporte, François-Xavier Esnault, and Philippe Guillemot, for their constant support and their help during the parabolic flight campaign.

[1] S. Bhaskaran, in the Telecommunications and Data Acquisition Progress Report 42-121 (NASA - Pasadena, California, United State, 1995), p. 54.

[2] J. Guena, M. Abgrall, D. Rovera, P. Laurent, B. Chupin, M. Lours, G. Santarelli, P. Rosenbusch, M. E. Tobar, R. Li, K. Gibble, A. Clairon, and S. Bize, Progress in atomic 
fountains at lne-syrte, IEEE Trans. Ultrason. Ferroelectrics Frequency Control 59, 391 (2012).

[3] P. Laurent, M. Abgrall, C. Jentsch, P. Lemonde, G. Santarelli, A. Clairon, I. Maksimovic, S. Bize, C. Salomon, D. Blonde, J. Vega, O. Grosjean, F. Picard, M. Saccoccio, M. Chaubet, N. Ladiette, L. Guillet, I. Zenone, C. Delaroche, and C. Sirmain, Design of the cold atom pharao space clock and initial test results, Appl. Phys. B 84, 683 (2006).

[4] F.-X. Esnault, D. Holleville, N. Rossetto, S. Guerandel, and N. Dimarcq, High-stability compact atomic clock based on isotropic laser cooling, Phys. Rev. A 82, 033436 (2010).

[5] F. Lienhart, S. Boussen, O. Carraz, N. Zahzam, Y. Bidel, and A. Bresson, Compact and robust laser system for rubidium laser cooling based on the frequency doubling of a fiber bench at $1560 \mathrm{~nm}$, Appl. Phys. B 89, 177 (2007).

[6] J. Weiner, V. S. Bagnato, S. Zilio, and P. S. Julienne, Experiments and theory in cold and ultracold collisions, Rev. Mod. Phys. 71, 1 (1999).

[7] W. Ketterle, A. Martin, M. A. Joffe, and D. E. Pritchard, Slowing and Cooling Atoms in Isotropic Laser Light, Phys. Rev. Lett. 69, 2483 (1992).

[8] H. Batelaan, S. Padua, D. H. Yang, C. Xie, R. Gupta, and H. Metcalf, Slowing of ${ }^{85} \mathrm{Rb}$ atoms with isotropic light, Phys. Rev. A 49, 2780 (1994).

[9] E. Guillot, P.-E. Pottie, and N. Dimarcq, Three-dimensional cooling of cesium atoms in a reflecting copper cylinder, Opt. Lett. 26, 1639 (2001).

[10] T. Hansch and A. Schawlow, Cooling of gases by laser radiation, Opt. Commun. 13, 68 (1975).

[11] D. J. Wineland, R. E. Drullinger, and F. L. Walls, RadiationPressure Cooling of Bound Resonant Absorbers, Phys. Rev. Lett. 40, 1639 (1978).
[12] J. Dalibard and C. Cohen-Tannoudji, Laser cooling below the doppler limit by polarization gradients: Simple theoretical models, J. Opt. Soc. Am. B 6, 2023 (1989).

[13] N. F. Ramsey, A molecular beam resonance method with separated oscillating fields, Phys. Rev. 78, 695 (1950).

[14] J. M. McGuirk, G. T. Foster, J. B. Fixler, and M. A. Kasevich, Low-noise detection of ultracold atoms, Opt. Lett. 26, 364 (2001).

[15] A. Clairon, P. Laurent, G. Santarelli, S. Ghezali, S. N. Lea, and M. Bahoura, A cesium fountain frequency standard: Preliminary results, IEEE. Trans. Instrum. Meas. 44, 128 (1995).

[16] G. J. Dick, J. D. Prestage, C. A. Greenhall, and L. Maleki, in 22nd Precise Time and Time Interval (PTTI) Applications and Planning Meeting (NASA - Pasadena, California, United State, 1990), p. 487.

[17] R. Lutwak, P. Vlitas, M. Varghese, M. Mescher, D. K. Serkland, and G. M. Peake, in Proceedings of the 2005 IEEE International Frequency Control Symposium and Exposition, 2005 (IEEE - Vancouver, British Columbia, Canada, 2005), p. 752.

[18] H. Hellwig, S. J. D. Halford Jr., and H. E. Bell, Evaluation and operation of atomic beam tube frequency standards using time domain velocity selection modulation, Metrologia 9, 107 (1973).

[19] R. Szmuk, V. Dugrain, W. Maineult, J. Reichel, and P. Rosenbusch, Stability of a trapped-atom clock on a chip, Phys. Rev. A 92, 012106 (2015).

[20] P. Yun, F. Tricot, C. E. Calosso, S. Micalizio, B. Francois, R. Boudot, S. Guérandel, and E. deClercq, HighPerformance Coherent Population Trapping Clock with Polarization Modulation, Phys. Rev. Appl. 7, 014018 (2017). 